\title{
A NEW LIBRARY PROGRAM FOR TEXTURE CALCULATIONS
}

F. WAGNER, C. ESLING and R. BARO

Laboratoire de Métallurgie Structurale, University of Metz, France

(Received February 18, 197.7)

Abstract: A new library program which allows the calculation and storage of the numerical tables necessary for a three-dimensional texture analysis is proposed. Its main characteristics are:

- possibility of selecting the values to be stored according to the desired microscopic and macroscopic symmetries as well as to the step of exploration of the pole figures;

- possibility of choosing the quantity of information to be stored for obtaining, in the further threedimensional analysis, a good agreement between the computing time and the memory space; and

- great precision of the stored values and short time of calculation due to the use of new and optimized algorithms.

\section{INTRODUCTION}

The computation chains used for the three-dimensional texture analysis (according to works of Bunge ${ }^{1}$ and of Roe ${ }^{2}$ ) generally call upon numerical data previously stored. ${ }^{3-5}$

The choice of the numerical data to be stored is essentially governed by two considerations:

- adjustment of these data to microscopic and macroscopic symmetries of the samples the texture of which will be studied, and adjustment to the step of exploration used for pole figures.

- adjustment of the number of stored data to the possibilities of the computer used for their later processing in analysis programmes. In fact, minimal times are required, and it is an advantage to have large data tables if there is enough space in the main storage unit. This is not necessarily true with very powerful computers whose times of access to peripheral units (disk or magnetic tape) and computing times necessary to restore the data tables must be compared. 
Finally, the precision of these values must be such that the errors which are necessarily introduced are very small in comparison with other causes of errors. ${ }^{6}$ This latter demand requires the use of highly performant algorithms, especially when the development into series of texture functions and intensity functions used for analysis'must reach high orders. It is, therefore, believed that to present a library program which best satisfies the aforementioned considerations would be of interest and usefulness.

\section{GENERALITIES AND DEFINITIONS}

The library program which we present is written in FORTRAN IV. The numerical data tables which are computed may be stored on a disk or a magnetic tape (sequential form). All the intermediate calculations are executed in double precision; final results are transferred to tables in simple precision form, and they are stored. One of the possibilities offered by our program is the establishment of a library which will be later called "standard library" and which is suited to the three-dimensional analysis chain proposed by Jura, Pospiech and Bunge. ${ }^{4}$

We define hereafter, according to the notations used by Bunge," the quantities which can be calculated and stored by our program.

i. Coefficients $Q_{\ell}^{m n}$ defined by:

$$
Q_{\ell}^{m n}=i^{m+n} P_{\ell}^{m n}(\pi / 2)
$$

where the functions $\mathrm{P}_{\ell}^{\mathrm{mn}}(\phi)$ are polynomials associated to the Jacobi polynomials ${ }^{8}, 9^{\ell}$ with

$$
\begin{aligned}
& \ell=2, \operatorname{LMAX}, 2 \text { and } 2 \leqslant \operatorname{LMAX} \leqslant 34 \text { (the third index is } \\
& \mathrm{m}=0, l, I D N \quad \text { IDN }=1 \text { or } 2 \text { the step of pro- } \\
& \mathrm{n}=0, \mathrm{~m}, \mathrm{IDN} \text { gression) }
\end{aligned}
$$

The limits fixed for LMAX are the same in all the following definitions. These $Q_{\ell}^{m n}$ coefficients, proposed by Bunge, lead to a good compromise between the storage space and the computing time necessary to build up the functions used in texture analysis. 9 They are always real. We use for their calculation a very performant algorithm which is described elsewhere. ${ }^{8}$

These coefficients which are calculated at the beginning of the program are used in all the following calculations.

ii. Functions $\overline{\mathrm{P}_{l}^{\mathrm{m}}}(\phi)$ defined by:

$$
\overline{\mathrm{P}_{\ell}^{\mathrm{m}}}(\phi)=i^{-\mathrm{m}}(2 \ell+1)^{1 / 2} \mathrm{P}_{\ell}^{\mathrm{mo}}(\phi)
$$

where $P_{\ell}^{m o}(\phi)$ always represents a polynomial associated with the Jacobi polynomial, with 


$$
\begin{aligned}
& \ell=2, \text { LMAX, } 2 \text { and } \operatorname{IDN}=1 \text { or } 2 \\
& \mathrm{~m}=0, \ell, \text { IDN } \quad 1.25^{\circ} \leqslant \Delta \phi \leqslant 90^{\circ} \\
& \phi=0,90^{\circ}, \Delta \phi
\end{aligned}
$$

These functions $\overline{\mathrm{P}_{\ell}^{\mathrm{m}}}(\phi)$ are calculated in our program by using their development into Fourier sums.

iii. Symmetrized harmonic functions $\dot{\mathrm{k}}_{l}^{\mu}\left(\mathrm{h}_{i}\right)$

with $\ell=2$, LMAX, 2

$\mu=1, M(l), 1$ where $M(l)$ is dependent on the

$$
h_{i}=\left\{\phi_{i}, \beta_{i}\right\}=\text { angles characterizing the normal }
$$
to the (hkl) plane required

These functions are linear combinations of the harmonic functions $k_{\ell}^{m}(\phi, \beta) .{ }^{7}$ The most general expression for them is:

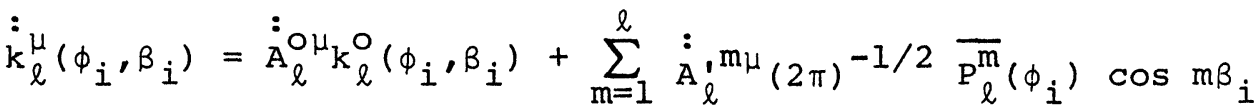

$$
\begin{aligned}
& +\sum_{m=1}^{\ell} \bar{A}_{\ell}{ }^{\prime m} \mu(2 \pi)^{-1 / 2} \overline{\mathrm{P}_{\ell}^{m}}\left(\phi_{i}\right) \sin \mathrm{m} \beta_{i}
\end{aligned}
$$

where the coefficients $\dot{:}_{l}^{o \mu}, \dot{:}_{l}^{\prime m \mu}$ and $\dot{A}^{\prime m}{ }_{l}^{m \mu}$ are symmetry coefficients, which are real $\left(\dot{\mathrm{A}}_{\ell}^{\circ}, \dot{\mathrm{A}}_{\ell}{ }^{\mathrm{m} \mu}\right)$ or imaginary $\left(\dot{\mathrm{A}}_{l}{ }^{\mathrm{m} \mu}\right)$.

They have very simple expressions except in the case of cubic symmetry which will be considered in a later section.

Our program allows the calculation of these functions $\dot{\mathrm{k}}_{\ell}^{\mu}\left(\mathrm{h}_{i}\right)$ for all the symmetry cases where the $\bar{A}_{\ell}{ }^{m}{ }^{m}$ coefficients are zero, i.e. for real $\dot{k}_{\ell}^{\mu}\left(h_{i}\right)$ functions, so that the definition expression can be written:

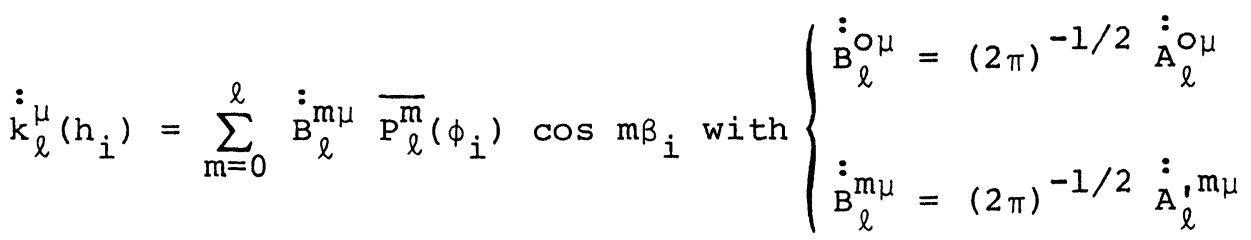

These symmetry cases correspond to all the Laue classes including the holoedry of crystalline systems except the triclinic system.

The case of the monoclinic system is rather different, because it requires an unusual choice of the microscopic reference axis ( $z$ axis in the mirror plane) for the definition 
(4) to be appropriate.

These functions can be calculated for 8 (hkl) planes at most in the case of cubic symmetry without data reading and/or for 12 (hkl) planes at most in the case of any other microscopic symmetry (except the triclinic). When a non-cubic symmetry is asked one has to specify the values of angles $\phi_{i}, \beta_{i}$ characterizing the $(\mathrm{hkl})$ planes required as input values (by card reader).

For the cubic symmetry the 8 possible (hkl) planes are: $(100),(110),(111),(102),(112),(122),(103),(113)$.

iv. Coefficients $a_{\ell}^{\prime m s}$

$$
a_{\ell}^{\prime m s}=\varepsilon(4 l+2)^{1 / 2} Q_{\ell}^{m s} Q_{\ell}^{\text {so }}
$$

where the coefficients $Q_{\ell}^{m n}$ are those defined in $i$. and where

$$
\begin{aligned}
& \varepsilon=(-1 / 2)^{\mathrm{m} / 2} \text { if } \mathrm{s}=0 \\
& \varepsilon=(-1)^{\mathrm{m} / 2} \text { if } \mathrm{m} \text { is even } \\
& \varepsilon=(-1)^{(\mathrm{m}-1) / 2} \text { if } \mathrm{m} \text { is odd }
\end{aligned}
$$

with $\ell=2$, LMAX, 2

$\mathrm{m}=0, \ell$, IDN IDN is an integer

$\mathrm{s}=0, \ell, 2$

These coefficients are real numbers.

v. Coefficients of cubic symmetry $\stackrel{\mathrm{B}}{\ell}_{\ell}^{\mathrm{m} \mu}$

with $\ell=2$, LMAX, 2

$$
\begin{aligned}
& \mathrm{m}=0, l^{\prime} 4 \\
& \mu=1, \quad \mathrm{M}(l), 1
\end{aligned}
$$

In the case of cubic symmetry, it is not possible to give a simple analytical expression which defines these coefficients. As a matter of fact, they are obtained during the search for linear combinations of harmonic functions $\mathrm{k}_{\ell}^{\mathrm{m}}(\phi, \beta)$ leading to the invariant functions $\dot{\mathrm{k}}_{\ell}^{\mu}\left(\mathrm{h}_{i}\right)$ for this symmetry. We use for the calculation of cubic symmetry coefficients $\dot{B}_{\ell}^{m} \mu$ a very efficient subroutine which was conceived after the work of Esling et al.10 concerning the use of projectors in the problems of symmetrisation.11

$$
\begin{array}{r}
\text { vi. Coefficients } a_{l}^{\prime m n s} \\
a_{\ell}^{\prime m n s}=\varepsilon Q_{\ell}^{m s} Q_{l}^{s n}
\end{array}
$$

$$
\begin{aligned}
& \text { where } \varepsilon=1 \text { if } s=0 \quad \text { with } l=2, \operatorname{LMAX}, 2 \\
& \varepsilon=2 \text { if } m+n \text { even } m=0, l \text {, IDM } \\
& \varepsilon=2 i \text { if } m+n \text { odd } \quad n=0, l \text {, IDN } \\
& s=0, l, 1
\end{aligned}
$$


For $s$ being different from zero and $m+n$ being odd these coefficients are imaginary numbers, $i . e .$, of the ib form. In these cases, the value $b$ alone is stored.

\section{ORGANIZATION}

Two main possibilities are offered by our program. The first one consists of the storing of the $Q_{l}^{m n}$ coefficients, the $\dot{\mathrm{k}}_{l}^{\mu}\left(\mathrm{h}_{\mathrm{i}}\right)$ symmetrized functions and the $\stackrel{\mathrm{B}}{\mathrm{m}}_{\ell}^{\mathrm{m}}$ coefficients if cubic symmetry is of interest.

The second one allows us to constitute a library which includes the $\overline{\mathrm{P}_{l}^{\mathrm{m}}}(\phi)$ functions, the $\mathrm{a}_{\ell}^{\prime \mathrm{ms}}$ and $\mathrm{a}_{\ell}^{\prime m n s}$ coefficients as well as the $\dot{\mathrm{k}}_{\ell}^{\mu}\left(\mathrm{h}_{\mathrm{i}}\right)$ functions, and eventually the $\dot{\mathrm{B}}_{\ell}^{\mathrm{m}} \mu$ coefficients.

In both cases, the LMAX value can be chosen, and so can be the step of progression of all the indices and of the angles included in the study.

The "standard library" answers a special choice of the different indices within the second possibility previously mentioned. Indeed, we have here LMAX $=34, \Delta \phi=5^{\circ}$, IDN $=2$ and $\operatorname{IDM}=4$, the microscopic symmetry required being the cubic symmetry.

Figure 1 shows the ordinogram of our library program. The three parameters ISTOK, IST, ISYM are respectively used to test the choice between the two main possibilities, the possible choice of the "standard library," and the desired microscopic symmetries. The parameter ISYM allows three options: cubic symmetry (ISYM = 1), a symmetry other than cubic (ISYM $=0)$, or cubic and another non-cubic symmetry (ISYM $=-1$ ). The listing of the library program is given in Annex $I$. Some information about the- necessary data cards (only one for "standard library") are given in Annex II. Lastly, explanations for reading again the stored values will be found in Annex III.

III. RESULTS

\section{Memory space used by the program}

The described library program requires $25 \mathrm{~K}$ words in the main storage unit, with words of 32 bits (4 bytes). This necessary memory space can be reduced by reducing the size of some arrays, when the maximum possibilities of the program are not used (for example LMAX < 34 or only one microscopic symmetry asked). The classical methods of overlay or of the storage of intermediate results outside the main memory also allow the use of this program with computers having less than $25 \mathrm{~K}$ words available. 


\section{Computing time}

The whole execution time of the program depends, of course, on the configuration of the computer used. Therefore, let us give for guidance only some computing times obtained with various equipments.

To create the "standard library" 13 minutes are necessary with an IBM $370 / 135$ computer, and 84 minutes with an IBM $370 / 11$

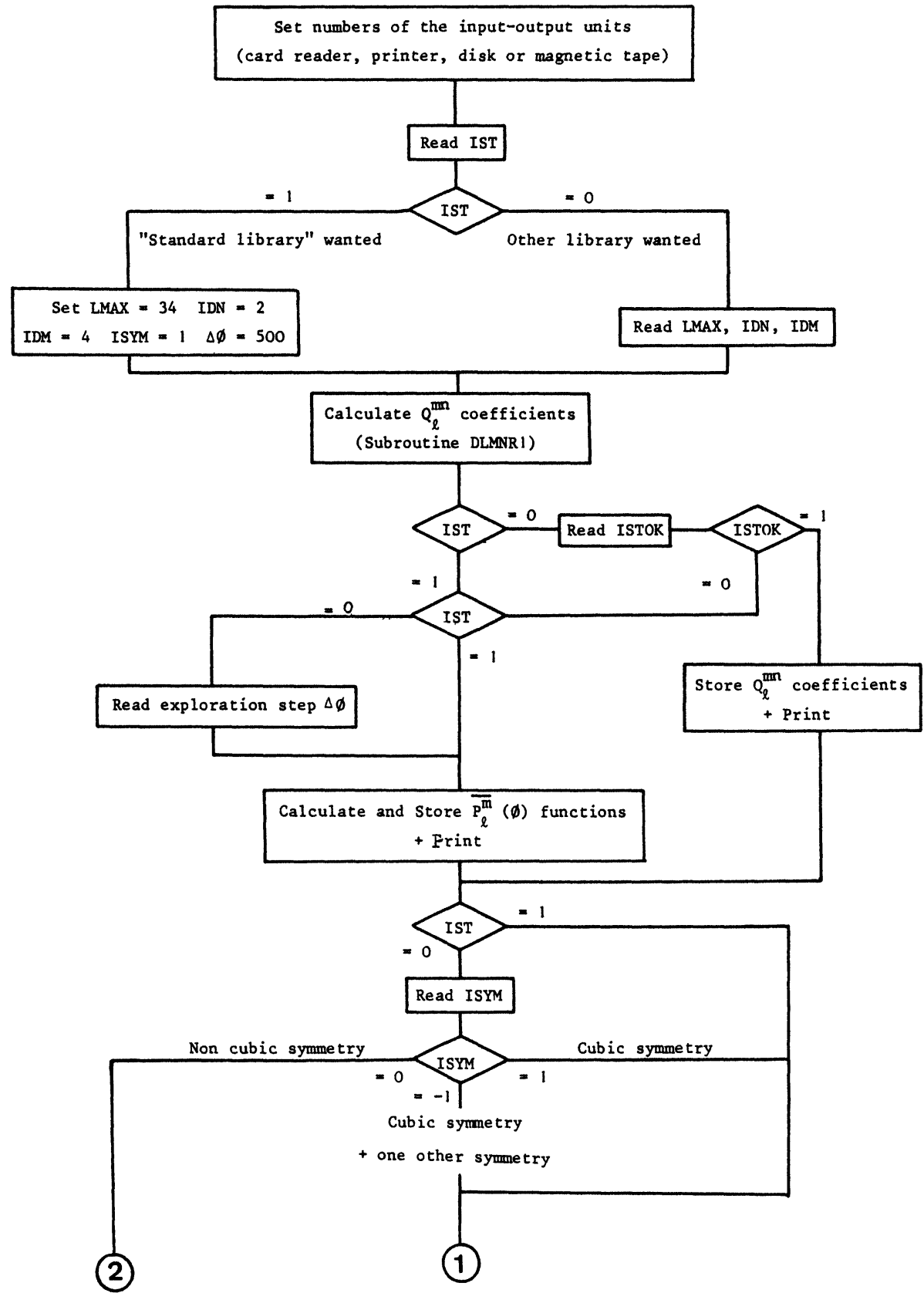




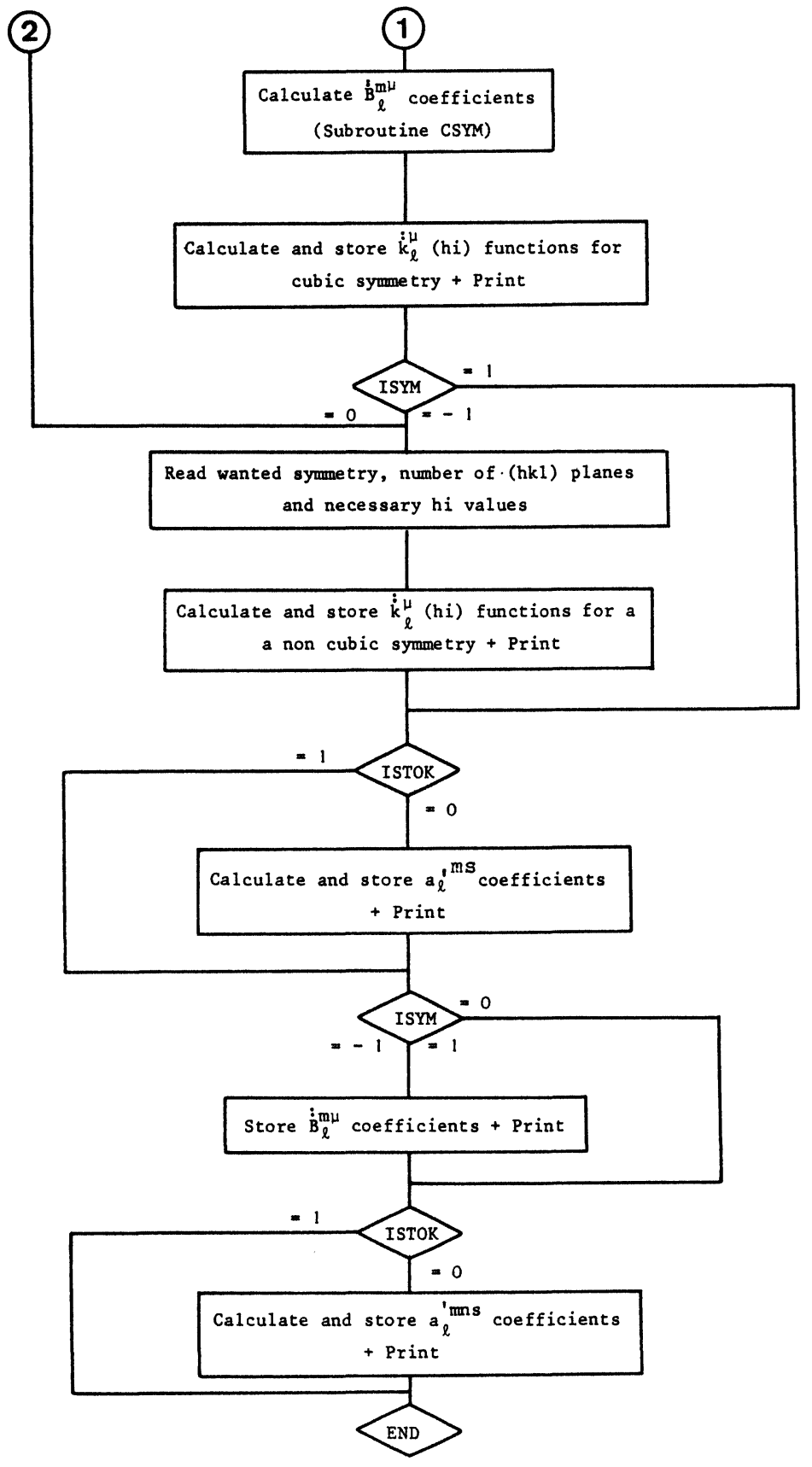

Figure 1. Ordinogram of the library program.

computer using virtual memory. By using overlay with a PDP $11 / 70$ computer having an available central memory of $32 \mathrm{~K}$ words of 16 bits, setting up a library where LMAX $=22$ takes 6.67 minutes. We must remark that the printing of all the results reduces considerably the speed of the carrying out 
of the program, the time necessary being approximately halved when the output instruction PRINT is cut out.

\section{Accuracy}

By using a computer which needs 32 bits to represent a real value in simple precision ( 64 bits in double precision) i.e. with about 7.2 decimal digits (16.8 in double precision), the error in the stored values is always smaller than one on the seventh significant digit.

A very detailed analysis of the errors on the $Q_{\ell}^{\mathrm{mn}}$ coefficients ${ }^{8}$ on which our program is based has shown that the calculation algorithm used gives at least 9 significant figures at order $l=34$. Therefore, it becomes obvious that for these coefficients as well as for the other stored values, the accuracy achieved is not determined by the calculation stages (in double precision), but by the size of the computer word ( 7.2 decimals in simple precision) used for the storage of the values.

\section{CONCLUSION}

The "library program" described here allows a coherent construction of all the data tables generally required for texture calculations. Thanks to the accuracy obtained for the stored values, it succeeds in reducing as much as possible the causes of errors, a constant subject of care in texture analysis. Because of its numerous possibilities, especially for that concerning symmetries, the development of the present program has taken these variables into consideration to enable the study of materials with diverse microscopic symmetries.

\section{ACKNOWLEDGEMENT}

We thank Mr. J. Pospiech for communicating programmes for three-dimensional texture analysis. We express our sincere gratitude to Monsieur P. Vayssiere, Director of the I.R.S.I.D. For allowing us to use the computer of his institute and to Monsieur J. C. Filler for constructive discussions about the programming problems.

\section{REFERENCES}

1. H. J. Bunge, Z. Metallkunde, 56, 872 (1965).

2. R. J. Roe, J. Appl. Phys., 36, 2024 (1965).

3. H. J. Bunge, Kristazl und Technik, 6, 429 (1971).

4. J. Jura, J. Pospiech and H. J. Bunge,, Texture, 1,201 (1974), and Metalurgia, 24, 111 (1976), Prace Komisji Metalurgiczno Odlewniczey Polska Akademia Nauk.

5. A. Clement and G. Durand, J. Appl. Cryst., 8, 589 (1975).

6. M. Humbert, Thèse de 3ème cycle, University of Metz (1976

7. H. J. Bunge, Mathematische Methoden der Texturanalyse, Akademieverlag Berlin, 1969, Chap. 11, pp. 215-261. 
8. C. Esling, et al., to be published.

9. H. J. Bunge, Kristall und Technik, 9, 939 (1974).

10. C. Esling, E. Bechler and R. Baro, to be published.

11. C. Esling, E. Bechler and R. Baro, Suppl. to Acta Crystal., A31, (1975).

\section{ANNEX I - LIBRARY PROGRAM}

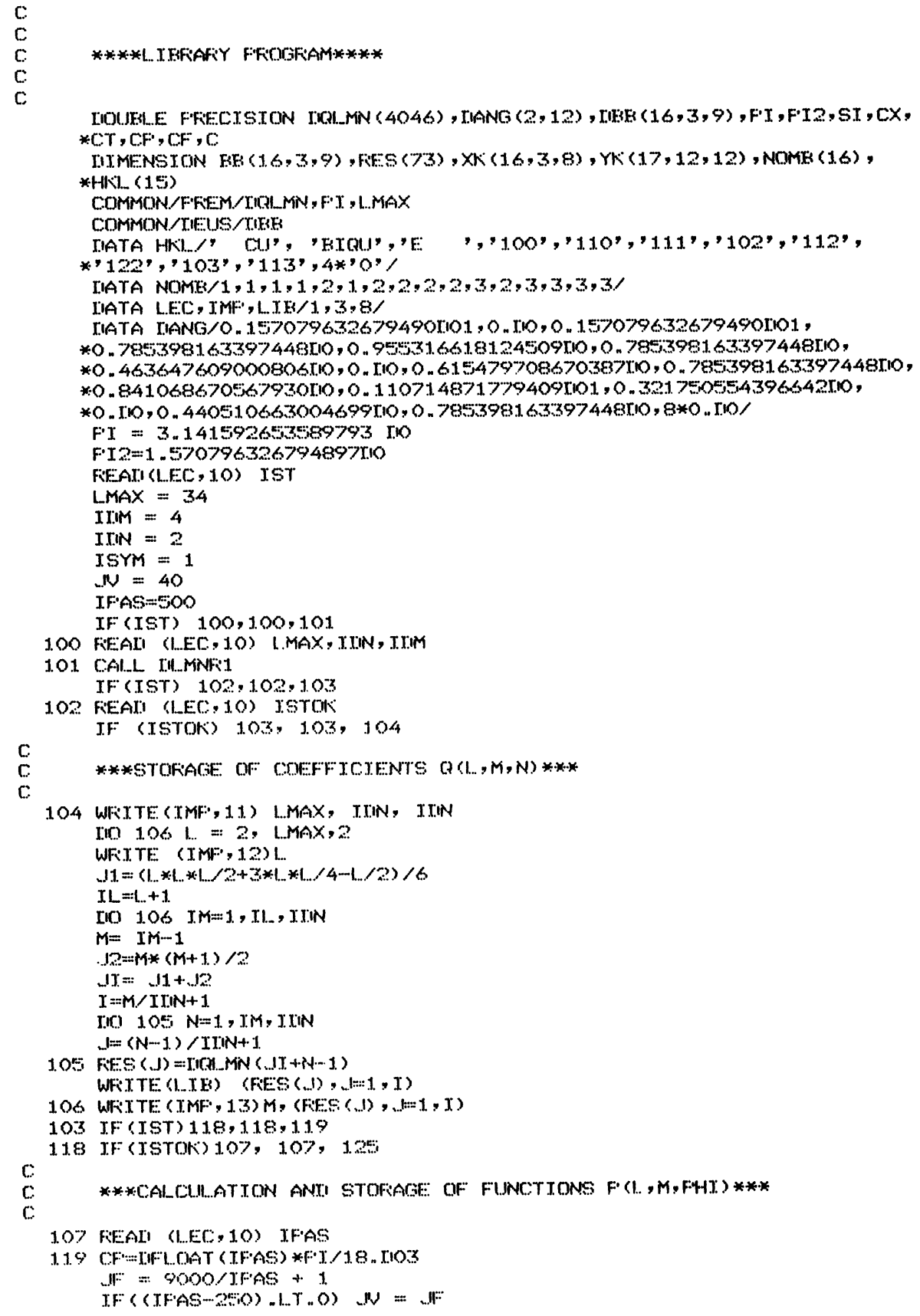




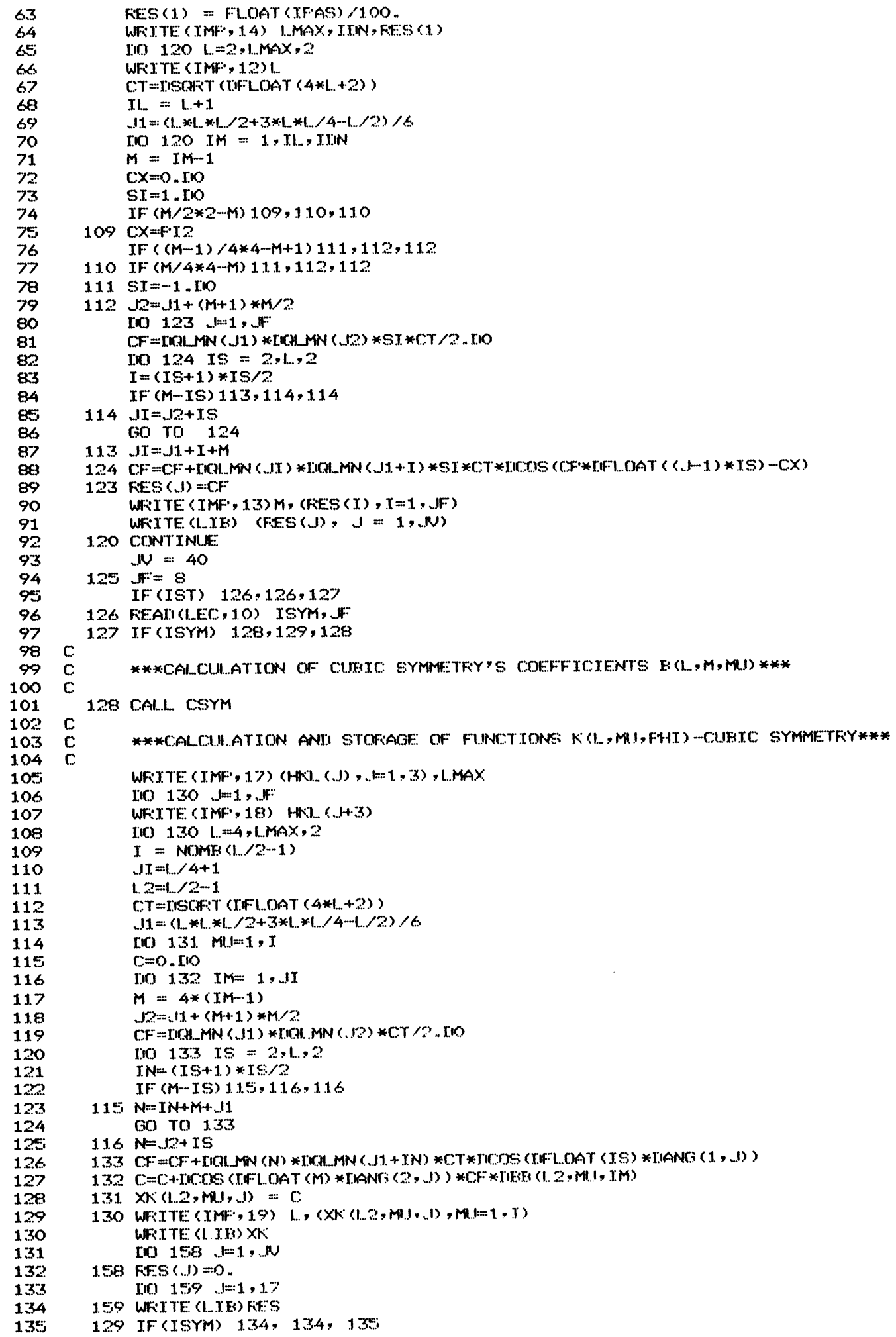




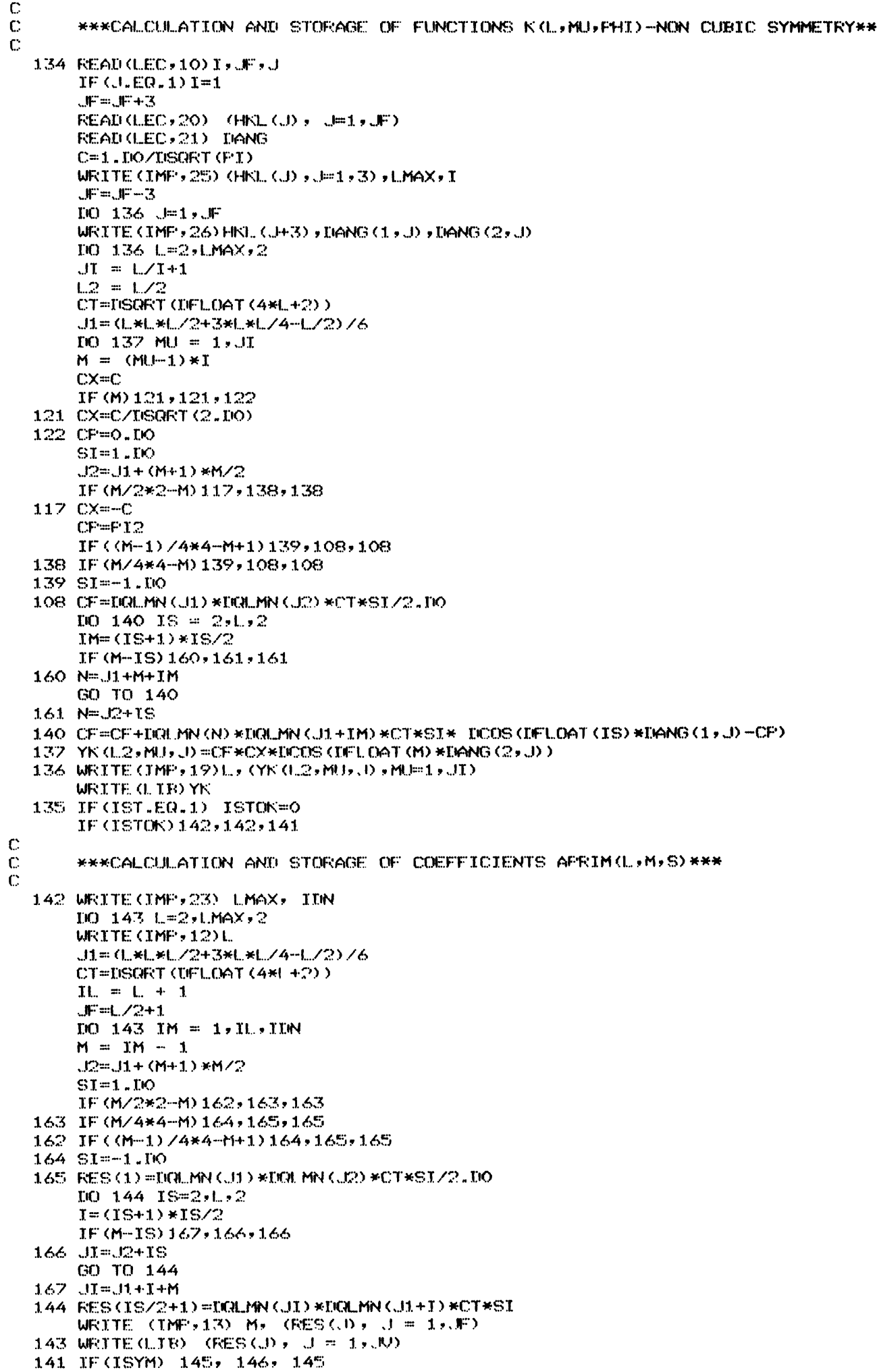




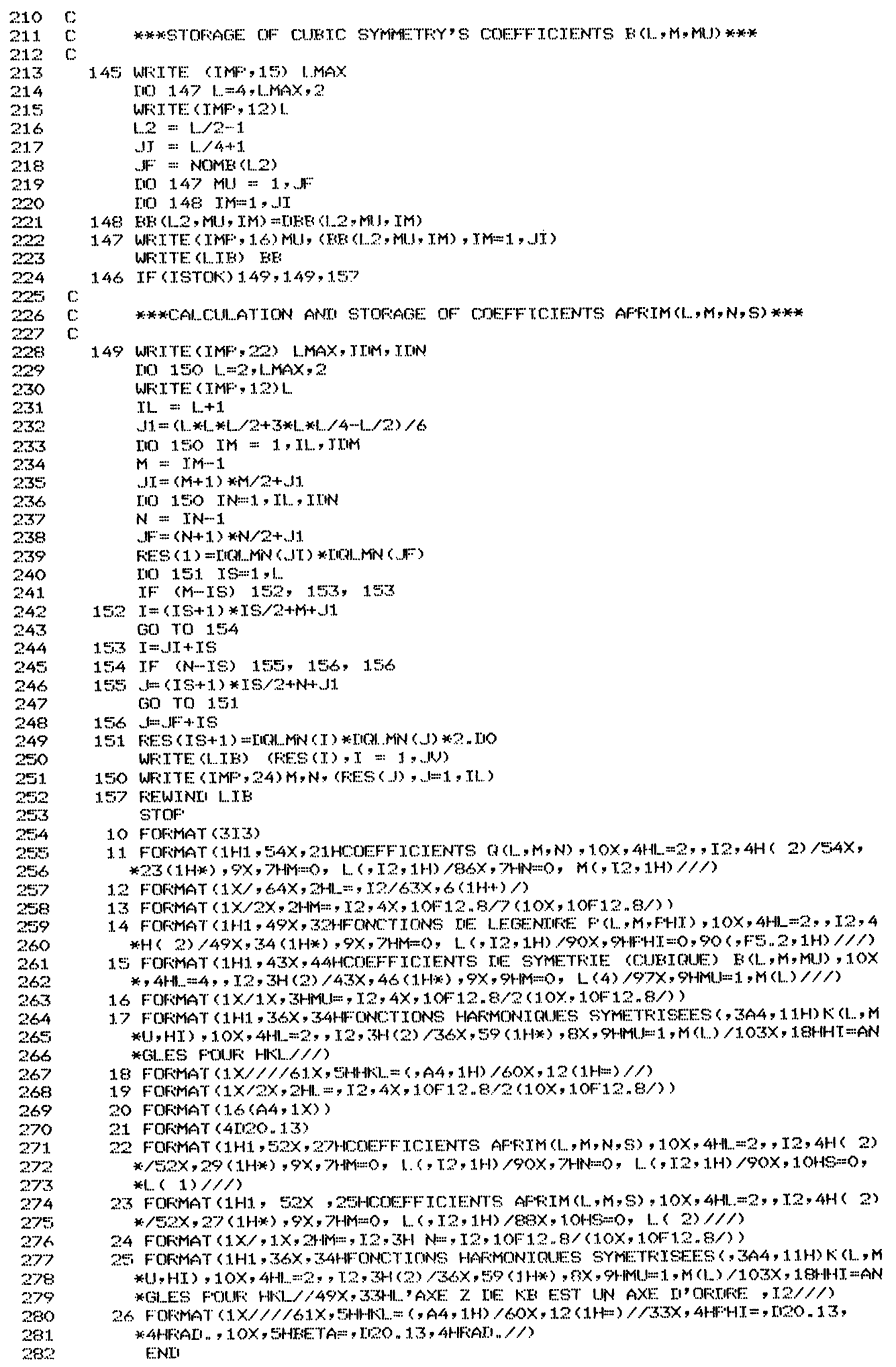




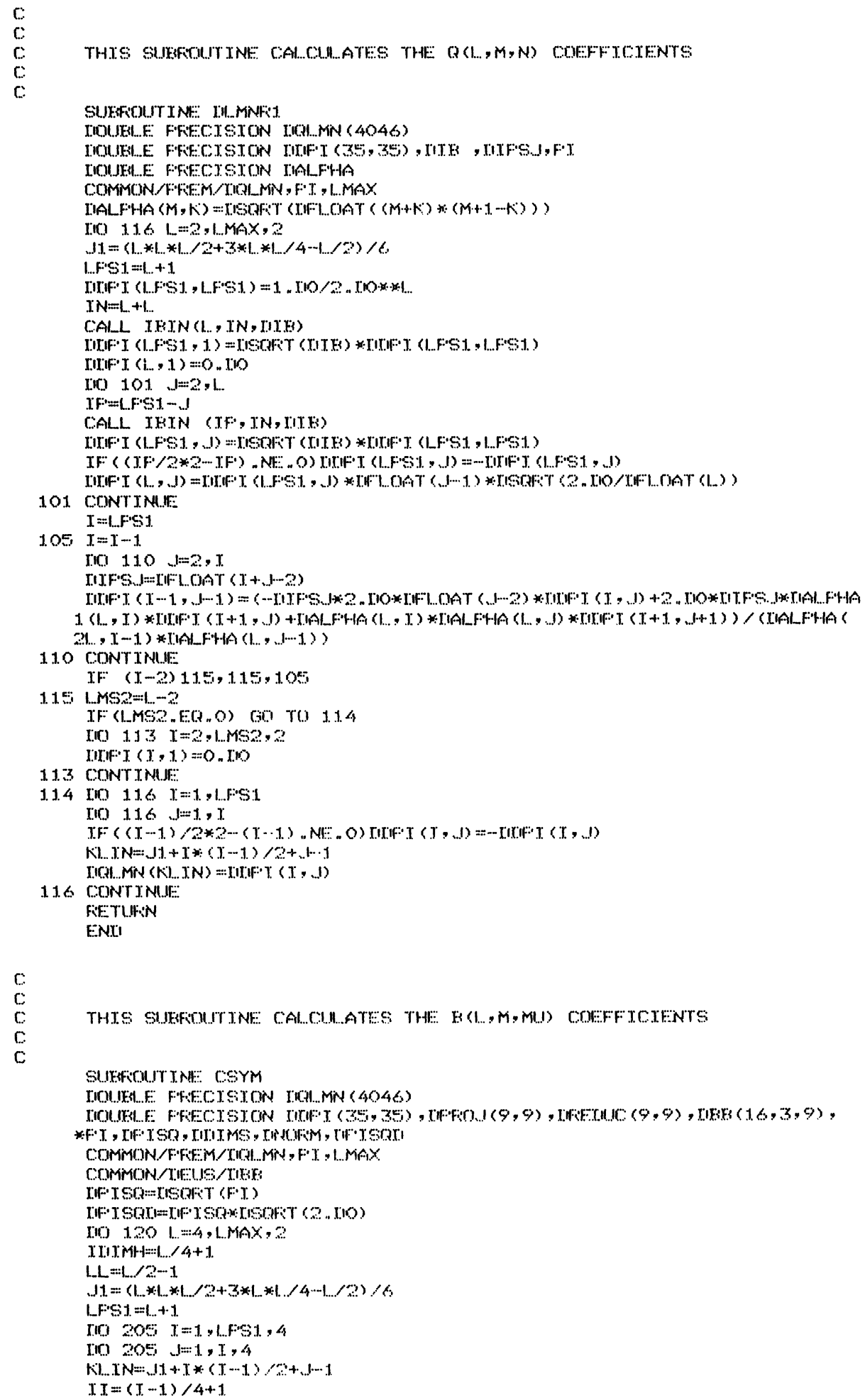




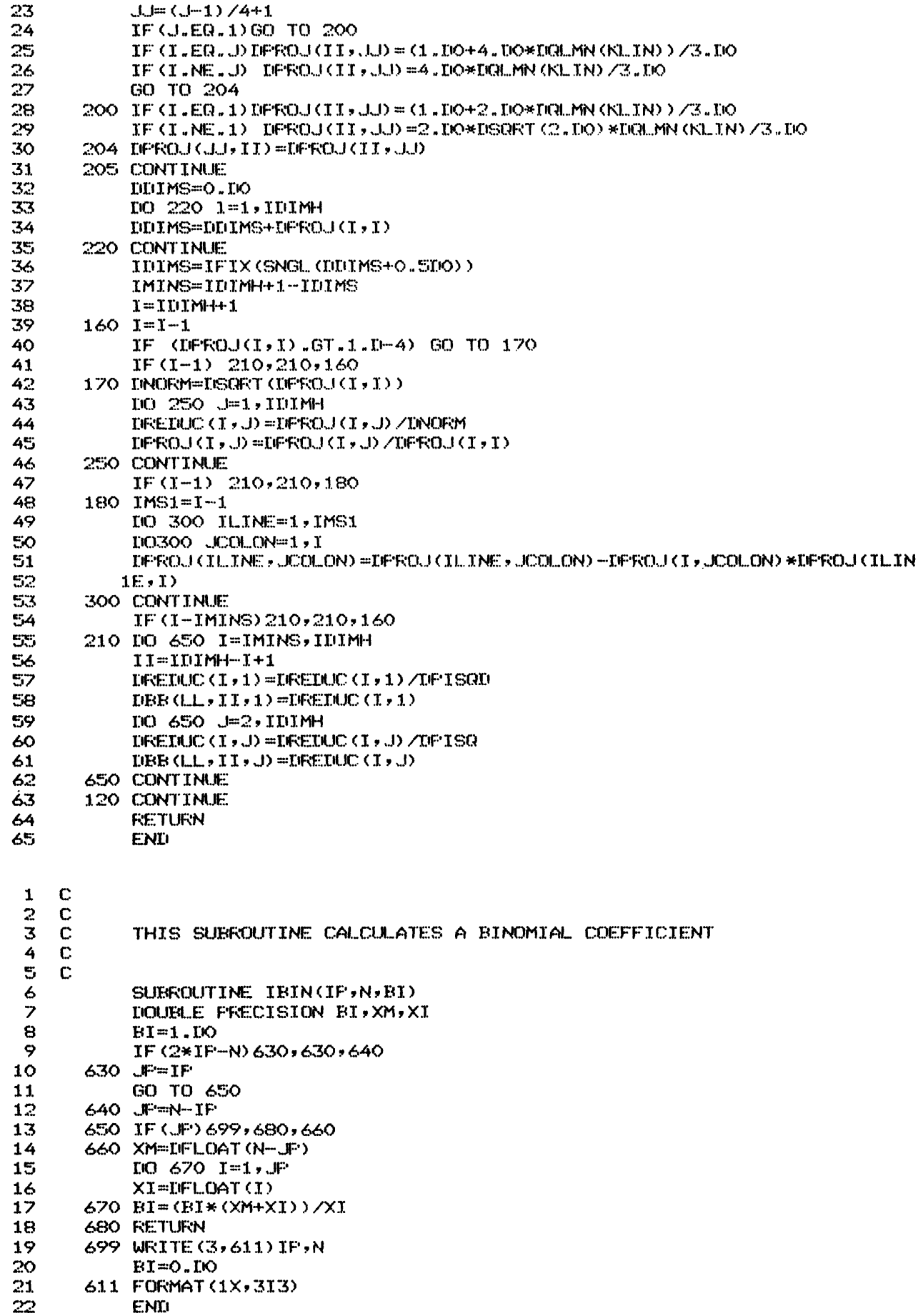


If the "standard library" is asked, only one data card is necessary with the value 1 in column 3 (value of parameter IST). To create any other library, the data cards must be presented in the order specified. All the cards settling the values of integer variables are read with a $3 I 3$ format.

1) 0 in column 3 (value of parameter IST)

2) Values of IMAX, IDN, IDM (see definitions in paragraph I and ordinogram for the signification of those parameters).

3) Value of ISTOK ( 0 or 1 according to the chosen option)

*4) Value of IPAS expressed in hundredths of degree. This card must be present only when ISTOK $=0$

5) Value of ISYM and JF where JF is the required number of (hkl) planes. When ISYM is equal to 0 or -1 (i.e. when a non-cubic symmetry is required) the following cards must be added:

*6) Values of $I, J F$ and $J$ where $I$ is the class of the highest symmetry axis (ex. 6 for hexagonal), JF is the number of (hkl) lattice planes required, $J$ is a parameter characterizing the monoclinic symmetry ( $J=1$ when this symmetry is asked, $J=0$ in the opposite case).

*7) Information about the symmetry asked and (hkl) lattice planes used. The reading is done with the help of a I5 (A4, IX) format. The data are classified in a 15 position array HKL (15). The first three array positions are reserved for the alphanumeric characters describing the required symmetry, the other twelve contain the (hkl) indices.

We give here an example of this data card:

$$
\begin{aligned}
& \begin{array}{lllll}
\text { column }: & 1 & 5 & 10 & 15
\end{array} \\
& \text { HEXA GONA LE } 10.011 .0 \ldots \text { and so on. }
\end{aligned}
$$

*8) Values of $\phi_{i}$ and $\beta_{i}$ angles characterizing the previously described (hkl) lattice planes. These values in radians must be given in double precision. They are read with a 4D20.13 format. Therefore, we have two pairs on each card; the maximum number of pairs is fixed to be 12 (i.e. 6 data cards here for angles $\phi_{i}$ and $\beta_{i}$ ).

Besides the "standard library" case, we must have at least 4 data cards. The stars (*) show the additional cards which must be added when some options are chosen. 


\section{CALLING THE STORED VALUES OF THE LIBRARY PROGRAM}

The library's values are stored in a sequential file on a magnetic band or a disk. Each time a WRITE statement is executed by the library program a logical record is created. The total number of records is, of course, dependent on the chosen library.

The writing is made in binary mode without format. So reading again the library must be made without format according to the order used to store it.

To reach a table of numerical data it is necessary to know the number of records which precede this table and to jump over them. For example, jumping over the 323 records containing the coefficients $Q_{\ell}^{m n}$ (with, in this case, $\ell=2$, LMAX, $2 ; m=0, \ell, 1 ; n=0, m, 1)$ can be achieved by the following statements:

$$
10 \| \begin{array}{lll}
\text { DO } 10 & I=1,323 & \\
\operatorname{READ} & (\mathrm{LIB}) & \begin{array}{l}
\text { where LIB specifies the logical } \\
\text { input-output unit for the library }
\end{array}
\end{array}
$$

For the coefficients $Q_{\ell}^{m n}, a_{l}^{\prime m s}$ and the functions $\overline{P_{l}^{m}}(\phi)$ the reading as well as the writing is made for fixed values of $l$ and $\mathrm{m}$, with an implicit DO statement involving the third "parameter" (index $n$, index $s$ and value of angle $\phi$ respectively). So, an array is filled up whose dimension must be at least equal to the number, let's say IMAX, of coefficients or functions obtained when the third "parameter" varies throughout. Then a READ statement will be of the form:

$$
\| \operatorname{READ}(\mathrm{LIB}) \quad(\mathrm{RES}(I), I=1, \operatorname{IMAX})
$$

where RES is an array in which the coefficients or functions are placed. This statement will be given in two loops in which $\ell(l=2$, LMAX, 2$)$ then $m(m=0, \ell$, IDN) are successively fixed.

For the coefficients $a_{\ell}^{\prime m n s}$ the READ statement is the same. However, it is made in three loops settling in this case $l$, $\mathrm{m}$ and $\mathrm{n}$.

The functions $\dot{\mathrm{k}}_{\ell}^{\mu}\left(\mathrm{h}_{i}\right)$ for cubic symmetry, $\dot{\mathrm{k}}_{\ell}^{\mu}\left(\mathrm{h}_{i}\right)$ for noncubic symmetry and the coefficients $\dot{B}_{l}^{m} \mu$ are placed within arrays with three indices (these arrays are named, in the librar: program, XK, YK and $\mathrm{BB}$ ). Each of these arrays is stored as a lump, i.e. with one WRITE statement, and therefore must be read again in the same way. For example, all the functions $\dot{\mathrm{k}}_{\ell}^{\mu}\left(\mathrm{h}_{i}\right)$ for cubic symmetry can be read with the statement: 
| READ (LIB) XK

The dimensions of these arrays are as follow:

|| $\operatorname{DIMENSION} \operatorname{XK}(16,3,8), \operatorname{YK}(17,12,12), \operatorname{BB}(16,3,9)$

The two first indices in the previous arrays correspond to the indices $l$ and $\mu$. The third is correlated with $h_{i}$ or $\mathrm{m}$ according as the functions $\dot{\mathrm{k}}_{\ell}^{\mu}\left(\mathrm{h}_{i}\right)$ or the coefficients $\dot{\mathrm{B}}_{\ell}^{\mathrm{m}} \mu$ are involved.

It is important also to note that, after the array $\mathrm{XK}$, 17 empty records are stored; this is only made so as to have a "standard library" which can be used directly in existing analysis programs.

For example we give hereafter a short program which allows to read again all the stored values in the "standard library" (let us remember that for this library LMAX $=34$, $\operatorname{IDN}=2, \operatorname{IDM}=4, \Delta \phi=5^{\circ}$ ).

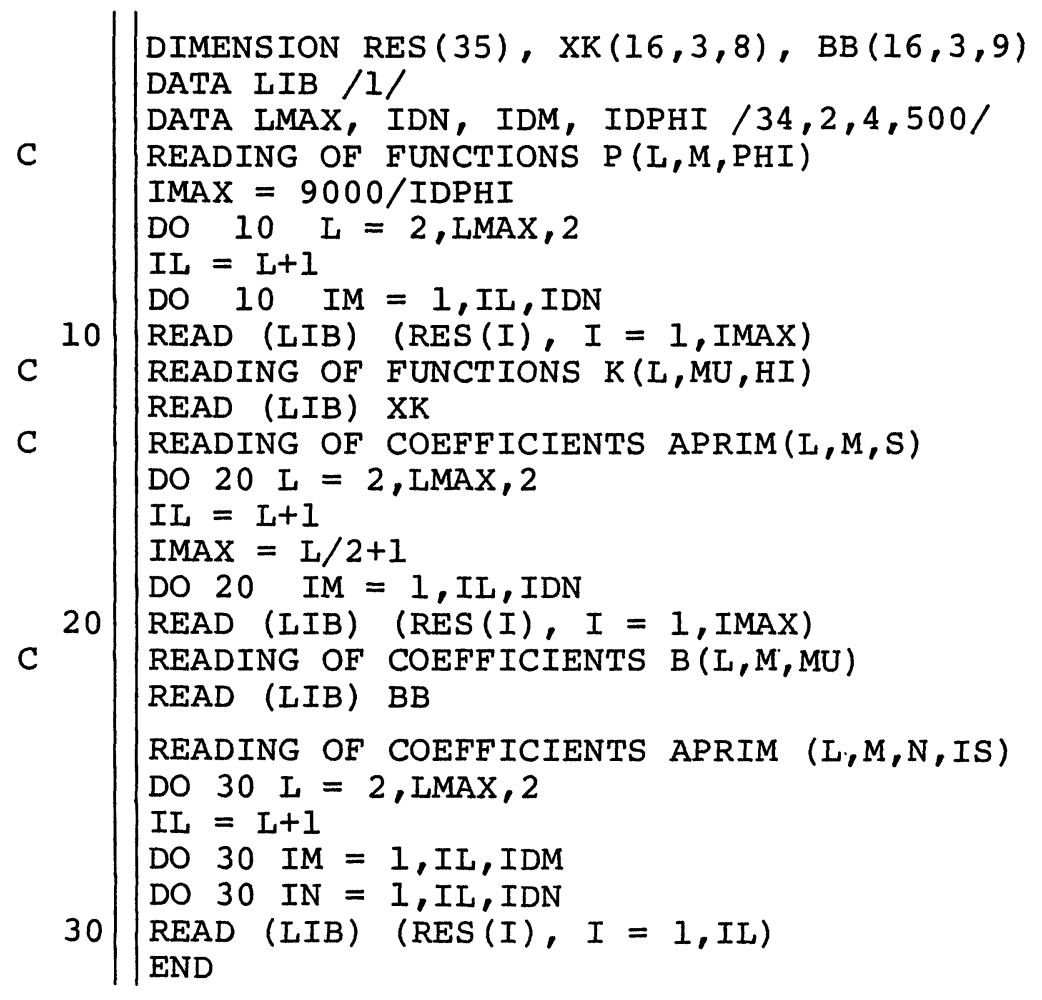

